\title{
Neutron-antineutron oscillation and baryonic majoron: low scale spontaneous baryon violation
}

\author{
Zurab Berezhiani ${ }^{1,2, a}$ \\ ${ }^{1}$ Dipartimento delle Scienze Fisiche e Chimiche, Università dell'Aquila, Via Vetoio, Coppito, 67100 L'Aquila, Italy \\ ${ }^{2}$ INFN, Laboratori Nazionali Gran Sasso, Assergi, 67100 L'Aquila, Italy
}

Received: 30 May 2016 / Accepted: 11 November 2016/ Published online: 23 December 2016

(C) The Author(s) 2016. This article is published with open access at Springerlink.com

\begin{abstract}
We discuss the possibility that baryon number $B$ is spontaneously broken at low scales, of the order of $\mathrm{MeV}$ or even smaller, inducing the neutron-antineutron oscillation at the experimentally accessible level. An associated Goldstone particle-baryonic majoron can have observable effects in neutron to antineutron transitions in nuclei or dense nuclear matter. By extending baryon number to an anomalyfree $B-L$ symmetry, the baryo-majoron can be identified with the ordinary majoron associated with the spontaneous breaking of lepton number, and it can have interesting implications for neutrinoless $2 \beta$ decay with the majoron emission. We also discuss the hypothesis that baryon number can be spontaneously broken by QCD itself via the six-quark condensates.
\end{abstract}

\section{Introduction}

There is no fundamental principle that can prohibit neutral particles as are the neutron or neutrinos to have a Majorana mass as envisaged long time ago by Majorana [1]. Nowadays the neutron is known to be a composite fermion having a Dirac nature conserving baryon number. As for the neutrinos, theorists prefer to consider them as Majorana particles though no direct experimental proofs for this were obtained yet (e.g. the neutrinoless double-beta decay). On the other hand, it is not excluded that the neutron $n$, along the large Dirac mass term $m \bar{n} n, m \approx 940 \mathrm{MeV}$, has also a small Majorana mass term $\epsilon_{n \tilde{n}} n^{T} C n+$ h.c. $=\epsilon_{n \tilde{n}} \bar{n} \tilde{n}+$ h.c., $\epsilon_{n \tilde{n}} \ll m$, which mixes the neutron and antineutron states (here $C$ is the charge conjugation matrix and $\tilde{n}=C \bar{n}^{T}$ is the antineutron field). This mixing induces the very interesting phenomenon of neutron-antineutron oscillation, $n \rightarrow \tilde{n}$, suggested a long time ago by Kuzmin [2]. The first theoretical scheme for the

\footnotetext{
a e-mail: zurab.berezhiani@ aquila.infn.it
}

$n-\tilde{n}$ oscillation was suggested in Ref. [3], followed by other types of models as e.g. in [4-6].

Clearly, the existence of the Majorana mass of the neutron would violate the conservation of baryon number $B$ by two units (analogously, Majorana masses for neutrinos violate lepton number $L$ by two units). If $B$ and $L$ were exactly conserved, the phenomena like proton decay, $n-\tilde{n}$ oscillation or neutrinoless double-beta decay would be impossible. Experimental limits on matter stability tell that $B$-violating processes must be very slow: lower bounds on the lifetime of the nucleons (and stable nuclei) land between $10^{30}-10^{34}$ years [7]. On the other hand, we have a strong theoretical argument that baryon number must be indeed violated in some processes. As was shown by Sakharov, $B$-violating processes which break also $\mathrm{CP}$ and which were out of equilibrium at some early cosmological epoch, can generate non-zero baryon number in the universe $[8,9]$. Without $B$-violation no primordial baryon asymmetry could be generated after inflation, the universe would remain baryon symmetric and thus almost empty of matter. (In modern theoretical scenarios, $B-L$ violation is indispensable and also sufficient [10].) One cannot exclude that primordial baryogenesis is related to the same $B$ and $B-L$ violating physics, containing CPviolating interactions of quarks, as also induces $n-\tilde{n}$ mixing as e.g. in the models of [3-6]. The relation of $n-\tilde{n}$ physics with P and CP violation was discussed in Ref. [11].

The structure of the standard model describing the known particles and their interactions nicely explains why the $B$ and $L$ violating processes are suppressed. Under the standard gauge group $G=S U(3) \times S U(2) \times U(1)$, the lefthanded (LH) quarks and leptons transform as iso-doublets $q_{L}=(u, d)_{L}, l_{L}=(v, e)_{L}$ while the right-handed (RH) ones are iso-singlets $u_{R}, d_{R}, e_{R} \cdot{ }^{1}$ As usual, one assigns a global lepton charge $L=1$ to leptons and a baryon charge $B=1 / 3$

\footnotetext{
1 For simplicity, in the following we omit the symbols L (left) and $\mathrm{R}$
} (right), the charge conjugation matrix $C$, and the internal gauge, spinor, 
to quarks, so that baryons composed of three valence quarks have a baryon number $B=1$.

However, $L$ and $B$ are not perfect quantum numbers. They are related to accidental global symmetries $U(1)_{L}$ and $U(1)_{B}$ possessed by the standard model Lagrangian at the level of renormalizable couplings (no renormalizable coupling can be written that could violate them). However, they can be explicitly broken by higher dimension (nonrenormalizable) operators suppressed by large mass scales which may be related to the scales of new physics beyond the standard model [12]. For example, grand unified theories (GUTs) introduce new interactions that transform quarks into leptons and thus induce effective four-fermion $(D=6)$ operators $\mathcal{O}_{6}=\frac{1}{M^{2}} q q q l$, etc. which lead to the proton decays like $p \rightarrow \pi e^{+}, p \rightarrow K v$ etc. These decay rates are suppressed by the GUT scale $M \geq 10^{15} \mathrm{GeV}$, which makes them compatible with the existing experimental limits [7].

It is well known that the lowest dimension $B-L$ violating operator $(D=5)$ is related to leptons and it violates the lepton number by two units $(\Delta L=2)$ [12]:

$$
\mathcal{O}_{5}=\frac{1}{M_{5}} l \phi l \phi+\text { h.c. }
$$

where $\phi$ is the Higgs doublet and $M_{5}$ is some large mass scale. After inserting the Higgs $\operatorname{VEV}\langle\phi\rangle=v \sim 100 \mathrm{GeV}$, this operator induces small Majorana masses for the neutrinos,

$m_{v} \sim \frac{v^{2}}{M_{5}} \sim\left(\frac{10^{14} \mathrm{GeV}}{M_{5}}\right) \times 0.1 \mathrm{eV}$

The experimental range of the neutrino masses, $m_{v} \sim 0.1 \mathrm{eV}$ or so, points toward $M_{5}$ being close to the GUT scale.

The neutron-antineutron mass mixing $\epsilon(\bar{n} \tilde{n}+$ h.c. $)$, violating baryon number by two units, can be related to the effective $D=9$ operators involving six quarks. In terms of the standard model fragments $u=u_{R}, d=d_{R}$, and $q=(u, d)_{L}$ these $\Delta B=2$ operators read

$\mathcal{O}_{9}=\frac{1}{\mathcal{M}_{9}^{5}}(u d d u d d+u d d q q d+q q d q q d)+$ h.c.

where $\mathcal{M}_{9}$ is some large mass scale. These operators can have different convolutions of the Lorentz, color, and weak isospin indices which are not specified. (Needless to say, the combination $q q$ in the second term in (5) must be in a weak isosinglet combination, $q q=\frac{1}{2} \epsilon^{\alpha \beta} q_{\alpha} q_{\beta}=u_{L} d_{L}$ where $\alpha, \beta=1,2$ are the weak $S U$ (2) indices, while in the third term $q q$ can be taken in a weak isotriplet combination as well.) More generally, having in mind that all quark families

Footnote 1 continued

and family indices whenever this will not cause ambiguities. Antiparticles will be termed $\tilde{q}, \tilde{u}, \tilde{d}$, etc. can be involved, these operators give rise to mixing phenomena also for other neutral baryons, e.g. oscillation of the hyperon $\Lambda$ into the antihyperon $\tilde{\Lambda}$.

If the scale $\mathcal{M}_{9}$ is taken of the order of the GUT scale, as one takes for the proton decaying operators $\mathcal{O}_{6}$ or for the leptonic operator $\mathcal{O}_{5}(1)$, the effects of $n-\tilde{n}$ mixing would become vanishingly small. On the other hand, the GUT scale is not really favored by the primordial baryogenesis. The latter preferably works at smaller scales, in the post-inflation epoch. An adequate scale for baryogenesis in the context of some $\Delta B=2$ models [5,6] can be as small as $\mathcal{M}_{9} \sim 1 \mathrm{PeV}$.

Taking into account that the matrix elements of operators $\mathcal{O}_{9}$ between the neutron states are of the order of $\Lambda_{\mathrm{QCD}}^{6} \sim 10^{-4} \mathrm{GeV}^{6}$, one can estimate (modulo the Clebsch coefficients $O(1))$ :

$\epsilon_{n \tilde{n}} \sim \frac{\Lambda_{\mathrm{QCD}}^{6}}{\mathcal{M}_{9}^{5}} \sim\left(\frac{10^{6} \mathrm{GeV}}{\mathcal{M}_{9}}\right)^{5} \times 10^{-25} \mathrm{eV}$.

The coefficients of matrix elements $\left\langle\tilde{n}\left|\mathcal{O}_{9}\right| n\right\rangle$ for different Lorentz and color structures of operators (3) were studied in Ref. [13] but we do not concentrate here on these particularities and take them as order 1 factors. In the presence of mixing $\epsilon_{n \tilde{n}}(\bar{n} \tilde{n}+$ h.c. $)$, the neutron mass eigenstates become two Majorana states $n_{+}=(n+\tilde{n}) / \sqrt{2}$ and $n_{-}=(n-\tilde{n}) / \sqrt{2}$, respectively, with the masses $m+\epsilon_{n \tilde{n}}$ and $m-\epsilon_{n \tilde{n}}$. The characteristic time of $n \rightarrow \tilde{n}$ oscillation in vacuum is related to their mass splitting, $\tau_{n \tilde{n}}=\epsilon_{n \tilde{n}}^{-1}$.

The direct experimental limit $\tau_{n \tilde{n}}>0.86 \times 10^{8} \mathrm{~s}(90 \%$ C.L.), obtained by a search of $n-\tilde{n}$ oscillation with cold neutrons freely propagating under the conditions of suppressed magnetic field [14], implies $\epsilon_{n \tilde{n}}<7.7 \times 10^{-24} \mathrm{eV}$. On the other hand, there are indirect limits from the nuclear stability: $n-\tilde{n}$ mixing inside the nuclei would destabilize the latter [15]. In fact, the operator (3) induces annihilation processes of two nucleons in two or more pions, $N N \rightarrow \pi$ 's, which transform the nucleus with atomic number $A$ into the nucleus with $A-2$ with emission of pions with total energy roughly equal to two nucleon masses. Interestingly, nuclear stability limits translated into the free $n-\tilde{n}$ oscillation time are not far more stringent than the direct experimental limit of Ref. [14]. In particular, the iron decay limit implies $\tau_{n \tilde{n}}>1.3 \times 10^{8} \mathrm{~s}(90 \%$ C.L.) [16], while the oxygen limit is about twice stronger, $\tau_{n \tilde{n}}>2.7 \times 10^{8}$ s $(90 \%$ C.L.) [17]. This settles the present upper limit on the $n-\tilde{n}$ mixing mass as $\epsilon_{n \tilde{n}}<2.5 \times 10^{-24} \mathrm{eV}$.

Thus, one can conclude that $n-\tilde{n}$ oscillation may test the underlying physics up to scales $\mathcal{M}_{9} \sim 1 \mathrm{PeV}$, having in mind that the experimental sensitivity can be improved by an order of magnitude, down to $\epsilon_{n \tilde{n}} \sim 10^{-25} \mathrm{eV}$. For the present status of $n-\tilde{n}$ oscillation and future projects for its search see e.g. Ref. [18].

One can envisage a situation when baryon number is broken not explicitly but spontaneously. In particular, one can 
consider a situation when baryon number is associated with an exact global symmetry $U(1)_{B}$, which is spontaneously broken by a complex scalar field $\chi$ with $B=2$. Spontaneous breaking of global $U(1)_{B}$ should give rise to a Goldstone boson $\beta$, which can be coined as the baryonic majoron, or baryo-majoron, in analogy to the leptonic majoron associated with the spontaneous breaking of global lepton symmetry $U(1)_{L}$ [19], which is widely exploited in neutrino physics.

In fact, spontaneous baryon violation in the context of $n-$ $\tilde{n}$ oscillation and the physics of the baryonic majoron was previously discussed in Ref. [20], in the context of the model [3]. Spontaneous $B$-violation was discussed also in Ref. [21], in terms of the operator $q q q l(B=1)$. The associated Goldstone boson was named a bary-axion, for respect of the electroweak anomaly of $U(1)_{B}$.

In this paper we discuss the possibility of spontaneous baryon violation at very low scales, order $\mathrm{MeV}$ or even less, in which case the baryo-Majoron can have observable consequences. Namely, it would induce nuclear decay via the Majoron emission, related to transition $n \rightarrow \tilde{n}+\beta$ in dense nuclear matter. Global baryonic number can be naturally extended to anomaly-free $B-L$. The spontaneous breaking of the latter must be relevant also for the neutrino Majorana masses. $^{2}$ In this way, the baryonic and leptonic majorons become in fact the same particle, just the majoron. In this context, we briefly discuss implications for leptonic sector as e.g. neutrinoless two-beta decay with the majoron emission and astrophysical implications of the neutrino decay. At the end, we also discuss a rather unusual possibility when baryon number is broken by six-quark condensates $\langle u d d u d d\rangle$ and its possible implications.

\section{Seesaw for neutron-antineutron mixing}

The contact (nonrenormalizable) $L$ and $B$ violating terms (1) and (3) can be induced in the context of UV-complete renormalizable theories after decoupling of some heavy particles. In particular, the leptonic operator (1) can be induced in the context of seesaw mechanism by introducing the Yukawa couplings $\phi \bar{N} l+$ h.c. which involve the heavy gauge singlet fermions $N_{(R)}$, the so-called right-handed neutrinos, with large Majorana mass terms, $M_{N} N^{2}+$ h.c., explicitly violating $L$ by two units. Then at lower energies $E \ll M_{N}$, the operator (1) emerges after integrating out the heavy neutrinos $N$ and, modulo the Yukawa constants, one gets $M_{5} \sim M_{N}$.

One can also discuss a simple seesaw-like scenario for the generation of $\Delta B=2$ terms (3). Let us introduce a gauge singlet Weyl fermion (or fermions) $\mathcal{N}_{(R)}$, a sort of heavy "RH neutron", and a color-triplet scalar $S$, with mass

\footnotetext{
2 One could consider global $B-L$ as a limit of a local gauge symmetry $U(1)_{B-L}$ when its gauge coupling constant is vanishingly small [22].
}

$M_{S}$, having precisely the same gauge quantum numbers as the right-handed down-quark $d_{(R)}$. Consider the Lagrangian terms

$S u d+S q q+S^{\dagger} d \mathcal{N}+M_{\mathcal{N}} \mathcal{N}^{2}+$ h.c.

where $q q$ in the second term is in a weak isosinglet combination, $q q=\frac{1}{2} \epsilon^{\alpha \beta} q_{\alpha} q_{\beta}=u_{L} d_{L}$ where $\alpha, \beta=1,2$ are the weak $S U(2)$ indices (we omit the charge conjugation matrix $C$ and Yukawa constants $\sim 1$ ). Baryon number is explicitly violated by the Majorana masses $M_{\mathcal{N}}$. Then, at energies $E \ll M_{S}, M_{\mathcal{N}}$, operators (3) are induced via integrating out the heavy states $S$ and $\mathcal{N}$. Thus, modulo the Yukawa constants in (5), one obtains

$\mathcal{M}_{9}^{5} \sim M_{S}^{4} M_{\mathcal{N}}$

From the model point of view, the scale $\mathcal{M}_{9} \sim 1 \mathrm{PeV}$, accessible via $n-\tilde{n}$ oscillation, may correspond to a "democratic" choice when $M_{\mathcal{N}} \sim M_{S} \sim 1 \mathrm{PeV}$. However, it can be obtained in different situations, namely by taking lighter $S$ and heavier $\mathcal{N}$ (e.g. $M_{S} \sim 10 \mathrm{TeV}$ and $M_{\mathcal{N}} \sim 10^{14} \mathrm{GeV}$ ), or heavier $S$ and lighter $\mathcal{N}$ (e.g. $M_{S} \sim 10^{7} \mathrm{GeV}$ and $M_{\mathcal{N}} \sim 100$ GeV).

In the above estimations, the mass scales $M_{N}$ and $M_{\mathcal{N}}$ are in principle independent parameters. In fact, the "heavy neutrino" $N$ and "heavy neutron" $\mathcal{N}$ cannot be the same particle. Otherwise its exchange would induce also operators like $u d d v$ with low cutoff scale which would induce unacceptably fast proton decay. If $N$ and $\mathcal{N}$ are assumed to be gauge singlets, then they can be divided by some discrete symmetries. This can be e.g. a $Z_{2}$ symmetry changing the sign of leptons, $l \rightarrow-l, e \rightarrow-e$, and $N \rightarrow-N$, while all other fields remain invariant. Equally, one can consider a baryonic $Z_{2}$ symmetry $q, u, d \rightarrow-q, u, d$ and $\mathcal{N} \rightarrow-\mathcal{N}$. Notice that these symmetries forbid also dangerous Yukawa couplings $S^{\dagger} q l$, which together with the couplings $S u d$ and $S q q$ would induce too fast proton decay.

Alternatively, one can consider $N$ as a weak isotriplet and $\mathcal{N}$ as a color octet, in which case no mixed mass terms may exist between $N$ and $\mathcal{N}$ states. For $\mathcal{N}$ being a color octet the scalars $S$ can be taken as color anti-sextets, in which case also the problematic couplings $S^{\dagger} q l$ will be automatically eliminated. The exchange via color octet $\mathcal{N}$ would generate operators $(u d d)_{8}(u d d)_{8}$ with $(u d d)_{8}$ in a color-octet combination. Via a Fierz transformation, exchanging $d$ states from the left and right brackets in this operator, we see that its matrix element will contribute to $n-\tilde{n}$ mixing.

In the context of supersymmetry, such operators can easily be obtained via $R$-parity breaking terms $u_{A} d_{B} d_{C}(B \neq C)$ in the superpotential, where $A, B, C$ are the family indices. Taking e.g. a superpotential term $u d s$ involving the up, down, and strange RH supermultiplets, one obtains the couplings 
analogous to $S u d+S^{\dagger} d \mathcal{N}$ of (5) with $S$ being the strange squark and $\mathcal{N}$ being a gluino with a Majorana mass $M_{\mathcal{N}}$. In fact, the gluino may have flavor-changing coupling between quark and squark states, namely between $d$-quark and $s$ squark. Needless to say, in this scheme somewhat bigger mixing mass would be generated for hyperons, between $\Lambda$ and $\tilde{\Lambda}$, via flavor diagonal gluino coupling between $s$-quark and $s$-squark. However, $\Lambda-\tilde{\Lambda}$ mixing is much more difficult to experimentally detect (though it maybe more efficient in the dense nuclear matter in the neutron stars where hyperons can emerge as natural occupants). In any case, $\Lambda-\tilde{\Lambda}$ mixing would also induce nuclear instability via two nucleon annihilation processes with kaon emission, $N+N \rightarrow K+K$ etc.

Let us consider now a situation when baryon number is broken not explicitly but spontaneously. Namely, let us modify the seesaw Lagrangian (5) as follows:

$S u d+S q q+S^{\dagger} d \mathcal{N}+\chi \mathcal{N}^{2}+$ h.c.,

where we prescribe $B=-2 / 3$ to scalars $S$ and $B=-1$ to extra fermions $\mathcal{N}$, and introduce a complex scalar field $\chi$ with $B=2$. In this way, all couplings in (7) respect the exact global symmetry $U(1)_{B}$. The non-zero VEV $\langle\chi\rangle=V_{\chi}$ spontaneously breaks the baryon number and induces the Majorana mass $M_{\mathcal{N}} \sim V_{\chi}$. Hence, the operator $\mathcal{O}_{9}$ emerges after the spontaneous baryon violation as shown on Fig. 1, with $n-\tilde{n}$ mixing parameter $\epsilon$ inversely proportional to the baryon symmetry breaking scale $V_{\chi}$ :

$\epsilon_{n \tilde{n}} \sim \frac{\Lambda_{\mathrm{QCD}}^{6}}{M_{S}^{4} M_{\mathcal{N}}} \sim\left(\frac{10^{30} \mathrm{GeV}^{5}}{M_{S}^{4} V_{\chi}}\right) \times 10^{-25} \mathrm{eV}$.

The scale $V_{\chi}$ can be related also to the breaking of lepton number if one extends global symmetry $U(1)_{B}$ to $U(1)_{B-L}$ and considers the following seesaw Lagrangian:

$\phi \bar{N} l+\chi^{\dagger} N^{2}+$ h.c.

In this way, after $U(1)_{B-L}$ breaking we obtain

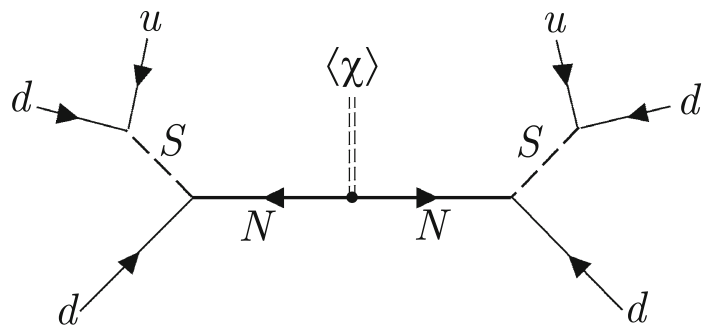

Fig. 1 Diagram generating $n-\tilde{n}$ mixing via exchange of $\mathcal{N}$ state which gets a large Majorana mass $M_{\mathcal{N}} \sim\langle\chi\rangle$ after $U(1)_{B}$ symmetry breaking $m_{v} \sim \frac{v^{2}}{M_{N}} \sim\left(\frac{10^{14} \mathrm{GeV}}{V_{\chi}}\right) \times 0.1 \mathrm{eV}$.

Since the neutrino masses favor the scale $V_{\chi} \sim 10^{14} \mathrm{GeV}$, according to the estimation (8) one can obtain $\epsilon_{n \tilde{n}}>10^{-25}$ $\mathrm{eV}$, potentially accessible in the search of $n-\tilde{n}$ oscillation, if the color scalars $S$ have masses in the range $M_{S} \sim 10 \mathrm{TeV}$; this is within the experimental reach for the new accelerators and perhaps also for the LHC. ${ }^{3}$

Here the following remark is in order. Although the direct limits on the heavy color scalars tolerate $M_{S} \sim 1 \mathrm{TeV}$, their exchange induces the effective operators $\left(g_{q q} / M_{S}\right)^{2}(\bar{q} q \bar{q} q)$ involving light quarks $q=u, d$, where $g_{q q}$ is the Yukawa constant of the coupling $S q q$ (or $S u d$ ) in (7). These contact terms are conventionally parameterized as $\left(2 \pi / \Lambda^{2}\right)(\bar{q} q \bar{q} q)$. The current limits on the compositeness scale $\Lambda$ are already above $10 \mathrm{TeV}$; namely, the recent LHC limits [24] yield $\Lambda>12 \mathrm{TeV}$ or $\Lambda>17.5 \mathrm{TeV}$ depending on their sign, i.e. whether these operators have destructive or constructive interference with the QCD processes. Hence, these bounds translate into the limits $M_{S} / g_{q q}>4.8 \mathrm{TeV}$ or $M_{S} / g_{q q}>7.0$ $\mathrm{TeV}$.

Constraints from the flavor violation can be generically stronger but they are rather model dependent. The couplings of scalar $S$ with light quarks, $S u d$, will not induce flavor violating transitions as $\bar{s} d \rightarrow \bar{d} s$ leading to $K-\bar{K}$ mixing etc. However, if the couplings involving heavier quarks are also present, with constants of order one, then the latter transition can be induced by box diagrams which could bring the limits on $M_{S}$ above $100 \mathrm{TeV}$. However, one can envisage some clever possibilities for suppressing these transitions without fine tunings of the Yukawa constants. Let us give one simple example, making use of chiral horizontal symmetry $S U(3)_{L} \times S U(3)_{R}$ under which the LH quarks $q=(u, d)_{L}$ of three generations transform as a triplet of $S U(3)_{L}$, the RH down quarks $d_{R}$ transform as a triplet of $S U(3)_{R}$, while the RH up quarks $u_{R}$ as well as the extra fermions $\mathcal{N}$ transform as anti-triplets of $S U(3)_{R}$. The Yukawa couplings for the fermion masses can be induced by the operators $\frac{\Phi_{u}}{M} \phi \bar{u}_{R} q_{L}$ and $\frac{\Phi_{d}}{M} \tilde{\phi} \bar{d}_{R} q_{L}$ introducing the flavon fields in mixed representations of $S U(3)_{L} \times S U(3)_{R}$, $\Phi_{u} \sim(\overline{3}, \overline{3})$ and $\Phi_{d} \sim(\overline{3}, 3)$ having large VEVs with hierar-

\footnotetext{
3 The masses of $N$ and $\mathcal{N}$ states are induced by the same scalar $\chi$ and thus they are $\sim V_{\chi}$. However, for guaranteeing proton stability, these states should be distinguished by other quantum numbers, as e.g. the leptonic and/or baryonic $Z_{2}$ symmetries discussed above. Let us also mention an interesting link between seesaw mechanisms for the generation of the neutrino and neutron Majorana masses: in parallel to the usual leptogenesis scenario [23] due to the heavy neutrino decays $N \rightarrow l \phi$ producing lepton number which then induces baryon number via $B-L$ conserving sphaleron effects [10]. Also baryogenesis can take place via the CP-violating effects in $\mathcal{N}$ decays mediated via colored scalar $S, \mathcal{N} \rightarrow \bar{d} S$ and $S \rightarrow \bar{u} \bar{d}$, which can directly produce the baryon number of the universe.
} 
chical and misaligned structures, in line with the mechanisms discussed in Refs. [25-28]. As for the states $\mathcal{N}$, they can get the Majorana masses via the operator $\frac{\left(\Phi_{u}^{\dagger} \Phi_{d}\right)_{A B}}{M} \mathcal{N}^{A} \mathcal{N}^{B}$, $A, B=1,2,3$ being the indices of $S U(3)_{R}$. In this case the couplings $S q q$ are forbidden while $S u d=S u^{A} d_{A}$ are diagonal in the flavor basis. As a result, the box diagrams induced by exchange of the $S$ scalar will have GIM suppression in the mass eigenstate basis, which will make $M_{S} \sim 10 \mathrm{TeV}$ perfectly compatible with the flavor violation limits.

Spontaneous breaking of global $U(1)_{B}$ and $U(1)_{L}$ gives rise to Goldstone bosons, baryo-majoron or lepto-majoron. ${ }^{4}$ These two can be the same particle, simply a majoron, once the global symmetry is promoted to $U(1)_{B-L}$. However, in practice a very large scale of symmetry breaking renders such majoron(s) unobservable experimentally and without any important astrophysical consequences. In the following section we discuss models where the global symmetry breaking scale can be rather small, $<1 \mathrm{MeV}$ or less, in which case the majoron interactions with the neutron as well as with neutrinos could have observable experimental and astrophysical consequences.

\section{Low scale seesaw model}

Is it possible to build a consistent model in which baryon number, or $B-L$, spontaneously breaks at rather low scales, in which case the majoron couplings to the neutrinos and to the neutron can be accessible for the laboratory search? This can be obtained by a simple modification of the above considered model.

Let us modify the Lagrangian terms (7) by introducing, along with the Weyl fermion $\mathcal{N}$ with $B=-1$, also another Weyl spinor $\mathcal{N}^{\prime}$ with $B=1$. These two together can form a heavy Dirac particle with a large mass $M=M_{D}$. The relevant Lagrangian terms now read

$S u d+S q q+S^{\dagger} d \mathcal{N}+M_{D} \mathcal{N} \mathcal{N}^{\prime}+\chi \mathcal{N}^{2}+\chi^{\dagger} \mathcal{N}^{\prime 2}+$ h.c.

Both $\mathcal{N}$ and $\mathcal{N}^{\prime}$ are coupled to the scalar $\chi$ (with $B=2$ ) and get the Majorana mass terms from the VEV of the latter, $\langle\chi\rangle=V_{\chi}$. We assume $V_{\chi}$ to be much less than $M$ and $M_{S}$. In this way, the diagram shown in Fig. 2, after integrating out the heavy fermions, induces $D=10$ operators invariant under $U(1)_{B}$ :

\footnotetext{
4 In reality, in the frames of the standard model both of these symmetries have electroweak anomalies and thus these majorons will get tiny masses of non-perturbative origin. However, $U(1)_{B-L}$ is free of anomalies and the corresponding Goldstone particles would exactly keep their masses. In any case, the tiny masses of the majoron will have no observable physical or astrophysical implications.
}
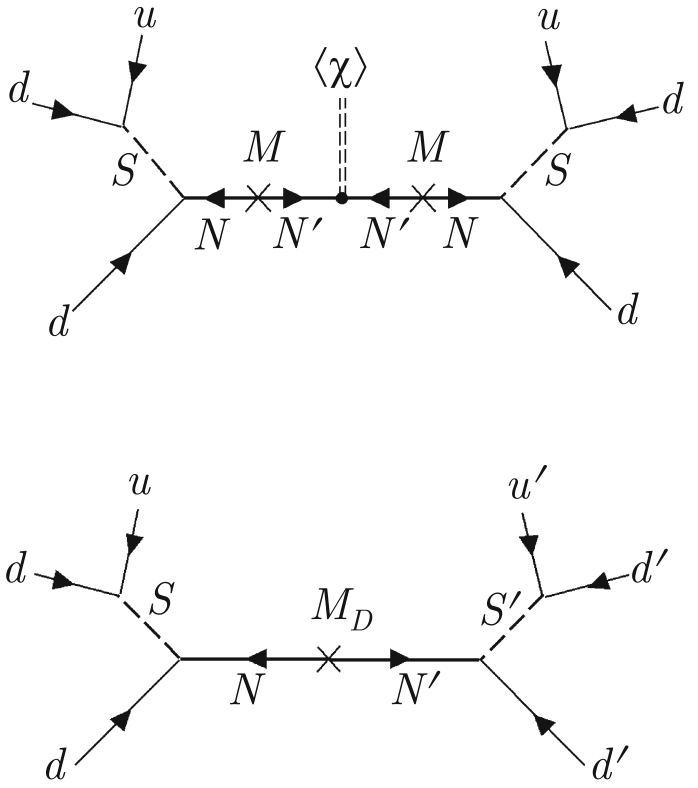

Fig. 2 Upper diagram generates $n-\tilde{n}$ mixing in a low scale model via exchange of the heavy Dirac fermion with mass term $M=M_{D}$, with insertion of the VEV $\langle\chi\rangle$. In the presence of mirror sector containing the twin quarks $u^{\prime}, d^{\prime}$ connected to $\mathcal{N}^{\prime}$, lower diagram generates $n-n^{\prime}$ mixing via only the Dirac mass term, without insertion of $\chi$ field

$\mathcal{O}_{10} \sim \frac{\chi^{\dagger}}{M_{D}^{2} M_{S}^{4}}(u d d u d d+u d d q q d+q q d q q d)+$ h.c.

Thus, at low energies these operators reduce to the neutron Yukawa couplings with the scalar $\chi$,

$Y_{n} \chi^{\dagger} n^{T} C n+$ h.c.

with the coupling constant

$Y_{n} \sim\left(\frac{\Lambda_{\mathrm{QCD}}^{3}}{M_{D} M_{S}^{2}}\right)^{2} \sim\left(\frac{10^{13} \mathrm{GeV}^{3}}{M_{D} M_{S}^{2}}\right)^{2} \times 10^{-30}$.

Once $U(1)_{B}$ is broken by the $\operatorname{VEV}\langle\chi\rangle=V_{\chi}$, the neutronantineutron mixing emerges as

$\epsilon_{n \tilde{n}}=Y_{n}\langle\chi\rangle \sim\left(\frac{10^{26} \mathrm{GeV}^{6}}{M_{D}^{2} M_{S}^{4}}\right)\left(\frac{V_{\chi}}{1 \mathrm{MeV}}\right) \times 10^{-24} \mathrm{eV}$.

Hence, if we take $M_{S} \sim 10 \mathrm{TeV}$ and $M_{D} \sim 100 \mathrm{TeV}$, or $M_{S} \sim 100 \mathrm{TeV}$ and $M_{D} \sim 1 \mathrm{TeV}$, then $\epsilon_{n \tilde{n}} \sim 10^{-24} \mathrm{eV}$ would require $V_{\chi} \sim 1 \mathrm{MeV}$ or so.

Low scale (but explicit) baryon number violation was suggested in Ref. [5], in a model which was mainly designed for inducing neutron-mirror neutron oscillation $n-n^{\prime}$ where the 
mirror neutron $n^{\prime}$ is the mass degenerate twin of the neutron which belongs to hypothetical mirror world, a parallel gauge sector with a particle content identical to that of the ordinary particles which is related to ordinary sector via parity transformation (for reviews, see e.g. [29-31]). Our model in fact generalizes the mechanism of Ref. [5] for the case of spontaneous $U(1)_{B}$ violation. In this case $\mathcal{N}$ and $\mathcal{N}^{\prime}$ states should be treated symmetrically: their Yukawa coupling constants with $\chi$ and $\chi^{\dagger}$ in (11) should be equal, while in addition the terms should be included that couple $\mathcal{N}^{\prime}$ to $u^{\prime}, d^{\prime}$, and $S^{\prime}$ states, twins of $u, d$, and $S$ from the mirror sector. In this way, the lower diagram of Fig. 2 induces $n-n^{\prime}$ mixing with

$\epsilon_{n n^{\prime}} \sim \frac{\Lambda_{\mathrm{QCD}}^{6}}{M_{D} M_{S}^{4}} \sim\left(\frac{10^{21} \mathrm{GeV}^{5}}{M_{D} M_{S}^{4}}\right) \times 10^{-16} \mathrm{eV}$,

which corresponds to an $n-n^{\prime}$ oscillation time of $\tau_{n n^{\prime}} \sim 10 \mathrm{~s}$. Hence, $n-n^{\prime}$ mixing, which conserves a combined baryon number $\bar{B}=B-B^{\prime}$ between ordinary and mirror sectors, can be a dominant effect, while $n-\tilde{n}$ mixing which breaks $\bar{B}$ is suppressed by the ratio $V_{\chi} / M_{D}$ :

$\epsilon_{n \tilde{n}}=\frac{V_{\chi}}{M_{D}} \epsilon_{n n^{\prime}}$.

As a matter of fact, $n-n^{\prime}$ mixing can indeed be much larger than $n-\tilde{n}$. Existing experimental limits on the $n-n^{\prime}$ transition [32-36] allow the neutron-mirror neutron oscillation time to be less than the neutron lifetime, with interesting implications for astrophysics and particle phenomenology [5,37-43].

Let us discuss the physics of the baryo-majoron, $\beta$, Goldstone boson related to the spontaneous breaking of global $U(1)_{B}$ symmetry. Its coupling to neutrons emerges by the substitution $\chi=V_{\chi} \exp \left(i \beta / f_{B}\right)$ in (13). It is worth to notice that in general the VEV $\langle\chi\rangle=V_{\chi}$ and the majoron decay constant $f_{\beta}$ are independent parameters. Namely, if $U(1)_{B}$ is broken solely by the VEV of $\chi$ which emerges via a negative mass $^{2}$ term in its potential, then we get $f_{\beta}=\sqrt{2} V_{\chi}$. In this case we have $\chi=\left(V_{\chi}+\frac{\rho}{\sqrt{2}}\right) \exp \left(i \beta / f_{B}\right)$, where $\rho$ is the massive (Higgs) mode with a mass $\sim V_{\chi}$, which can be light and which will interact with the same Yukawa constants as the majoron. But in the generic case, when besides the scalar $\chi$ there are also some other scalars $\eta_{i}$, with baryon charges $B_{i}$, which get non-zero VEVs $\left\langle\eta_{i}\right\rangle=V_{i}$, for the majoron decay constant we have $\sqrt{2} f_{\beta}=\sqrt{4 V_{\chi}^{2}+\sum_{i}\left(B_{i} V_{i}\right)^{2}}>2 V_{\chi}$. For example, one can imagine a situation when $\chi$ itself has a large positive mass ${ }^{2}$ term, but its VEV is induced by nonzero VEV $V_{\eta}$ of a scalar $\eta$ with $B=1$, via the coupling term $\chi^{\dagger} \eta^{2}$ in the Higgs potential:

$$
\begin{aligned}
\mathcal{V}(\chi, \eta)= & M_{\chi}^{2} \chi^{\dagger} \chi+\frac{h}{2}\left(\chi^{\dagger} \chi\right)^{2}-m^{2} \eta^{\dagger} \eta+\frac{\lambda}{2}\left(\eta^{\dagger} \eta\right)^{2} \\
& +\mu\left(\chi^{\dagger} \eta^{2}+\text { h.c. }\right) .
\end{aligned}
$$

In the limit $\mu \rightarrow 0, \eta$ gets a $\operatorname{VEV}\langle\eta\rangle=V_{\eta}=\sqrt{m^{2} / \lambda}$ while $\langle\chi\rangle$ is vanishing. However, for $\mu \neq 0$, the non-zero VEV of $\chi$ is also induced, $V_{\chi} \approx \mu V_{\eta}^{2} / M_{\chi}^{2} \ll V_{\eta}$. In this case we have $f_{\beta} \approx V_{\eta} / \sqrt{2} \gg V_{\chi}$. For example, taking $m \sim 1$ $\mathrm{MeV}, \mu \sim 1 \mathrm{GeV}$ and $M_{\chi} \sim 10 \mathrm{GeV}$, we get $V_{\chi} \sim 10$ $\mathrm{eV}$ against $f_{\beta} \sim 1 \mathrm{MeV}$. Then, in view of Eqs. (14) and (15), for achieving the experimentally testable range for $n-\tilde{n}$ mixing, $\epsilon_{n \tilde{n}} \sim 10^{-25} \mathrm{eV}$ or so, we must have $Y_{n} \sim 10^{-24}$. For $M_{S} \sim 10 \mathrm{TeV}$, this would require $M_{D} \sim 1 \mathrm{TeV}$ or so.

In any case, the majoron $\beta$ is coupled non-diagonally between the $n$ and $\tilde{n}$ states, $i g_{n} \beta \bar{n} \gamma_{5} \tilde{n}+$ h.c., with the Yukawa constant $g_{n}=Y_{n} V_{\chi} / f_{\beta}$. As far as $\epsilon_{n \tilde{n}}=Y_{n} V_{\chi}$, we thus have the relations ${ }^{5}$

$g_{n}=\frac{\epsilon_{n \tilde{n}}}{f_{\beta}}, \quad Y_{n}=\frac{f_{\beta}}{V_{\chi}} g_{n}$.

Therefore, for $f_{\beta} \gg V_{\chi}$, the Yukawa coupling (13) of the scalar $\chi$ can be large, $Y_{n} \gg g_{n}$, while $\chi$ itself can be rather heavy, $M_{\chi} \gg 1 \mathrm{GeV}$. For example, in the context of the Lagrangian (18) we have $M_{\chi} \simeq \sqrt{\mu / V_{\chi}} f_{\beta}$.

In vacuum the transition $n \rightarrow \tilde{n}+\beta$ is suppressed as far as the masses of $n$ and $\tilde{n}$ are equal. However, in nuclei the neutron and antineutron have different effective potentials and thus a $n \rightarrow \tilde{n}$ transition with majoron emission becomes possible. (This phenomenon is rather similar to matter induced neutrino decay with majoron emission $[44,45]$.) The decay rate of the neutron in nuclei can be estimated as

$\Gamma(n \rightarrow \tilde{n} \beta)=\frac{g_{n}^{2}}{2 \pi} \Delta E_{n \tilde{n}}$,

where $\Delta E_{n \tilde{n}}=\left|U_{\tilde{n}}-U_{n}\right|$ is a typical energy budget for $n \rightarrow \tilde{n}$ transition (the average difference between the neutron and antineutron potentials inside the nucleus) which is typically of order $10 \mathrm{MeV}$ for lighter nuclei and it can reach several $100 \mathrm{MeV}$ for heavier nuclei. (Needless to say, if the massive component $\rho$ of the scalar $\chi$ is light, with the mass order $\mathrm{MeV}$, then the nuclear transition $n \rightarrow \tilde{n}+\rho$ is also allowed.) The produced antineutron promptly annihilates with other spectator nucleons producing pions, with total energy roughly equal to two nucleon masses.

However, experimentally it is difficult to distinguish $n \rightarrow$ $\tilde{n}+\beta$ decay from the nuclear decay due to annihilation of two

\footnotetext{
5 The following remark is in order. In general, the operators (12) do not respect parity and also violate $\mathrm{CP}$. Therefore, after taking the matrix element $\left\langle n\left|\mathcal{O}_{10}\right| \tilde{n}\right\rangle$, in addition the coupling $Y_{n} \chi^{\dagger} n^{T} C n+$ h.c. (13) there can emerge a coupling $X_{n} \chi^{\dagger} n^{T} C \gamma^{5} n+$ h.c., with generically complex constant $X_{n}$. After the VEV $\langle\chi\rangle$ is inserted, the mass term $\epsilon^{\prime} n^{T} C \gamma^{5} n$ can be rotated away and so only the term $\epsilon_{n \tilde{n}} n^{T} C n$ is relevant for $n-$ $\tilde{n}$ mass mixing [11], with $\epsilon_{n \tilde{n}}=V_{\chi} \sqrt{Y_{n}^{2}+X_{n}^{2}}$. However, the term $Y_{n}^{\prime} \chi^{\dagger} n^{T} C \gamma^{5} n$ will contribute the majoron couplings which will have a generic form $i g_{n} \beta \bar{n}\left(a+b \gamma_{5}\right) \tilde{n},|a|^{2}+|b|^{2}=1$. Unfortunately, the effects of $\mathrm{P}$ and $\mathrm{CP}$ violation in the majoron couplings can hardly be detectable in experiments.
} 
nucleons in two or more pions induced by $n-\tilde{n}$ oscillation, since the majoron takes only a small fraction of the energy. ${ }^{6}$ In the latter case, the nuclear disappearance rate per neutron can be estimated as [15]

$\Gamma_{n \tilde{n}} \simeq \frac{\epsilon_{n \tilde{n}}^{2} \Gamma_{\tilde{n}}}{\Delta E_{n \tilde{n}}^{2}+\left(\Gamma_{\tilde{n}} / 2\right)^{2}}$

where $\Gamma_{n} \sim 300 \mathrm{MeV}$ is the antineutron annihilation rate at nuclear densities. Let us remark also that, for heavier nuclei, with very large $\Delta E_{n \tilde{n}}$, the rate (21) decreases, while the rate of majoron induced decay (20) is proportional to $\Delta E_{n \tilde{n}}$ and so it becomes more effective for heavier nuclei.

The present limits on the $n-\tilde{n}$ transition lifetime of the neutron bound in nuclei are at the level of $10^{32}$ years. Namely, for iron one has $\Gamma_{n \tilde{n}}^{-1}<7.2 \times 10^{31}$ (90\% C.L.) [16] and for oxygen $\Gamma_{n \tilde{n}}^{-1}<1.9 \times 10^{32}(90 \%$ C.L.) [17]. For $n-\tilde{n}$ mixing, these limits, respectively, yield $\epsilon_{n \tilde{n}}<1.2 \times 10^{-24} \mathrm{eV}$ and $\epsilon_{n \tilde{n}}<2.5 \times 10^{-24} \mathrm{eV}$. As far as $n \rightarrow \tilde{n}+\beta$ decay is regarded, in view of Eq. (20) these limits translate into a bound of $g_{n}<10^{-31}$ or so.

Imagine now that in future experiments with large volume detectors the signal for $n \rightarrow \tilde{n}$ is found at the level of a transition lifetime of $10^{32}-10^{33}$ years. This could be interpreted via $n-\tilde{n}$ mixing with $\epsilon_{n \tilde{n}} \sim 10^{-24} \mathrm{eV}$, or via the decay $n \rightarrow \tilde{n}+\beta$ with $g_{n} \sim 10^{-31}$. However, the first possibility can be discriminated by the direct search of $n-\tilde{n}$ oscillation with free neutrons at the European Spallation Source (ESS) in which a sensitivity down to $\epsilon_{n \tilde{n}} \sim 10^{-25} \mathrm{eV}$ can be achieved [18]. Hence, if the result of the direct search at the ESS will be negative, then the positive result in nuclei can be interpreted via the majoron induced decay.

Now, if one promotes $U(1)_{B}$ symmetry to $U(1)_{B-L}$ in the context of a low scale model, $f_{\beta} \leq 1 \mathrm{MeV}$, the baryomajoron should be the same particle as the usual (leptonic) majoron. The Majorana masses of the neutrinos can be induced, as in the model suggested in Ref. [48], from the diagram shown in Fig. 3 via the following Lagrangian terms:

$\phi \bar{l} N+M N N^{\prime}+\chi N^{2}+\chi^{\dagger} N^{\prime 2}+$ h.c.

where $N, N^{\prime}$ are the fermion couples, analogous to $\mathcal{N}, \mathcal{N}^{\prime}$, with properly assigned lepton number (or better $B-L$ ) and they have large Dirac masses $M \sim M_{D}{ }^{7}$ Then after inte-

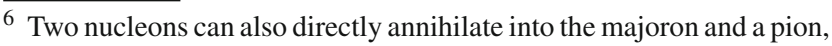
$N N \rightarrow \beta+\pi$, phenomenon resembling the neutrinoless $2 \beta$ decay with the majoron emission [46-48], though violating not lepton but baryon number. Since the majoron itself is not detectable, a characteristic signal in this case can be provided by the nuclear transition $A \rightarrow A-2$ with a single pion emission with energy $E \approx m_{n}$, while the majoron takes away the same energy. However, the branching ratio of this process is small with respect to multi-pion annihilation.

${ }^{7}$ For simplicity, we take their Dirac mass terms $M$ and $M_{D}$ parametrically of the same order. However, we recall that the "heavy neutrinos"
}

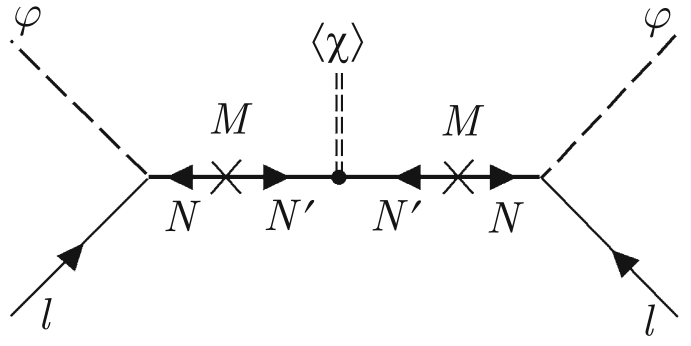

Fig. 3 Diagram generating the neutrino majorana masses in a low scale majoron model

grating out of the heavy states, one obtains the operator

$\mathcal{O}_{6} \sim \frac{\chi}{M_{D}^{2}} l \phi l \phi+$ h.c.,

which at lower energies results in the neutrino Yukawa couplings with the scalar $\chi$,

$Y_{\nu} \chi v^{T} C v+$ h.c.

with

$Y_{\nu} \sim \frac{v^{2}}{M_{D}^{2}} \sim\left(\frac{100 \mathrm{TeV}}{M_{D}}\right)^{2} \times 10^{-6}$

Then for the neutrino Majorana masses we have

$m_{\nu}=Y_{\nu}\langle\chi\rangle \sim\left(\frac{100 \mathrm{TeV}}{M_{D}}\right)^{2}\left(\frac{V_{\chi}}{1 \mathrm{MeV}}\right) \times 1 \mathrm{eV}$.

Taking into account also uncertainties in the Yukawa constants in (22), this estimate falls in the experimental mass range of neutrinos when $M_{D} \sim 100 \mathrm{TeV}$ and $V_{\chi}<1 \mathrm{MeV}$. For $M_{D} \sim 1 \mathrm{TeV}$, when $Y_{v} \sim 10^{-2}$, one needs $V_{\chi} \sim 10$ $\mathrm{eV}$. As we have shown above, this situation can be obtained when $V_{\chi}$ is induced by scalars $\eta$ via the Lagrangian (18) while $f_{\beta}=\sqrt{2} V_{\eta} \sim 1 \mathrm{MeV} .^{8}$

Footnote 7 continued

$N, N^{\prime}$ and "heavy neutrons" $\mathcal{N}, \mathcal{N}^{\prime}$ must be different particles distinguished by some discrete symmetry as e.g. $Z_{2}$ : otherwise their exchange would induce the operators like $u d d v$ with a low cutoff scale leading to dramatically fast proton decay.

8 The following remark is in order. Even if the scalar $\chi$ itself has a large mass $M_{\chi}$ and its small VEV is induced by $\eta$, the annihilation process of two neutrons in two antineutrinos can take place in the nuclei, with the rate $\Gamma(n n \rightarrow \bar{v} \bar{v})=\langle\sigma v\rangle n_{\text {nucl }} \sim Y_{n}^{2} Y_{v}^{2}\left(10 \mathrm{GeV} / M_{\chi}\right)^{2} \times 10^{-6} \mathrm{GeV}$, where $n_{\text {nucl }} \approx 1.2 \times 10^{38} \mathrm{~cm}^{-3}$ is the nuclear density. Confronting this with the experimental bounds on dinucleon decay, $\Gamma^{-1}(n n \rightarrow \bar{v} \bar{\nu})>$ $10^{30}$ years [7], we obtain an upper limit $Y_{n} Y_{v}\left(10 \mathrm{GeV} / M_{\chi}\right)<10^{-28}$ or so. In view of Eqs. (14) and (25), this translates into the bound $M_{D} M_{S}\left(M_{\chi} / 10 \mathrm{GeV}\right)^{1 / 4}>10^{7} \mathrm{GeV}^{2}$. The Lagrangian model (18) with $f_{\beta} \sim 1 \mathrm{MeV}$ and $V_{\chi} \sim 10 \mathrm{eV}$ corresponds to this limit for the benchmark values $M_{\chi} \sim 10 \mathrm{GeV}, M_{S} \sim 10 \mathrm{TeV}$, and $M_{D} \sim 1 \mathrm{TeV}$. 
In this situation, the majoron $\beta$ can have pretty large Yukawa couplings with the neutrinos, $g_{v}=m_{v} / f_{\beta}$ [48]. Hence, for $f_{\beta}<100 \mathrm{keV}$ one could have $g_{v}>10^{-6}$ or so, in the range of interest for searching the neutrinoless twobeta decay with the majoron emission $[46,47]$. The present experimental bound on the majoron coupling to $v_{e}$ reads $g_{v_{e}}<(0.8-1.6) \times 10^{-5}$ [49]. Analogous couplings with other neutrino flavors can bring about observable effects with interesting applications for astrophysics and cosmology as e.g. matter induced neutrino decay $[44,45,50-52]$ or matter induced decay of the majoron in two neutrinos [53], blocking of active-sterile oscillations in the early universe by the majoron field [54,55], etc. A detailed analysis of the astrophysical limits on the neutrino-majoron couplings can be found in [56].

A few words about cosmological limits. If the majoron coupling constants with neutrinos are large, majorons will come into equilibrium with neutrinos by reactions $v v \rightarrow \beta \beta$ etc. in the early universe before the neutrino decoupling $(T \sim$ $2 \mathrm{MeV}$ ) and their presence would contribute to the effective number of neutrinos as $\Delta N_{v}=4 / 7$, at the margin of current BBN bounds. For the low scale majoron with $f_{\beta} \sim V_{\chi} \leq$ $1 \mathrm{MeV}$, this value will be doubled by the contribution of the Higgs mode $\rho$, in evident contradiction with the BBN limits.

Confronting the majoron production rate $\Gamma_{\beta}=\sigma n_{\nu}$ with the Hubble parameter $H=1.66 g_{*}^{1 / 2} T^{2} / M_{P l}$, where $g_{*}=10.75$ is the effective number of particle degrees of freedom at the temperatures $T$ of few $\mathrm{MeV}, n_{v}=0.18 T^{3}$ is the equilibrium density for one neutrino species, and $\sigma=4 \times 10^{-3} \cdot g_{v}^{4} / T^{2}$ is the $v v \rightarrow \beta \beta$ reaction crosssection, with the Hubble rate $H$ at $T>2 \mathrm{MeV}$, one obtains the condition $g_{v}=m_{v} / f_{\beta}<3 \times 10^{-5}$. Thus, in view of the cosmological limits on neutrino masses, $m_{v}<0.3 \mathrm{eV}$, one can see that majorons do not come into equilibrium if $f_{\beta}>10 \mathrm{keV}$ or so.

Certainly, the scalar $\chi$ should come into equilibrium also in very early universe, $T>M_{D}$, by interactions with the heavy fermions $N, N^{\prime}$ etc. with large Yukawa couplings $\chi N N$ etc. in (22). However, it decouples at $T<M_{D}$, while neutrinos remain in equilibrium with all standard particle degrees of freedom with $g_{*}\left(T<M_{D}\right)=110$ or perhaps even more if there are new particles with masses less than $M_{D}$ as e.g. color scalars $S$. Therefore, at the BBN temperatures, $T \sim 1 \mathrm{MeV}$, the majorons will have a lower temperature than the neutrinos, $T_{\beta} / T_{v}=\left[g_{*}\left(T=M_{D}\right) / g_{*}(T=\right.$ $1 \mathrm{MeV})]^{1 / 3}>(110 / 10.75)^{1 / 3} \simeq 2.2$ so that two spin 0 states $\beta$ and $\rho$ together will count as $\Delta N_{\nu}=0.05$ extra neutrinos. The same applies to the case when $\chi$ is heavy, $M_{\chi}>$ few $\mathrm{GeV}$, but it Yukawa couplings to neutrinos $Y_{\nu} \chi \nu^{T} C \nu$ are large, say $Y_{v} \sim 10^{-2}$. In this case the majoron will decouple at the temperatures $T \sim M_{\chi}$. Therefore, the BBN limits can be fully respected.
Concluding this section, we have shown that in our model with low scale $B-L$ breaking, $f_{\beta} \leq 1 \mathrm{MeV}$, the majoron $\beta$ can have large enough Yukawa coupling to neutrons, in the range $g_{n} \sim 10^{-31}$, as well as to neutrinos, in the range $g_{v}>$ $10^{-7}$ or so. Therefore, such a scenario can be tested in large volume detectors searching for baryon violation via nuclear instabilities well as in the experiments testing the lepton number violation with the neutrinoless two-beta decays, etc.

However, there arises a naturalness issue questioning how $B-L$ can be broken by the VEVs of elementary scalars $\leq 1 \mathrm{MeV}$, at least five orders of magnitude less than the electroweak scale $M_{Z} \sim 100 \mathrm{GeV}$, without invoking fine tuning. Namely, in the absence of other fields like $\eta$, when scalar $\chi$ gets a VEV from its own Higgs potential, it should have a negative $M_{\chi}^{2}$ of the order of $1 \mathrm{MeV}^{2}$. In the context of the potential $\mathcal{V}(\chi, \eta(18)$ when the VEV of $\chi$ is induced by the VEV of $\eta$, the latter should have negative $m^{2} \sim 1 \mathrm{MeV}^{2}$.

The way out is to relate $f_{\beta}$ with some compositeness scale. Imagine that the scalar $\chi$, having positive mass term $M_{\chi}^{2} \chi^{\dagger} \chi$ with $M_{\chi}>1 \mathrm{GeV}$, has the Yukawa couplings $\chi \bar{Q} Q$ with the quark-like states of some hidden sector with a confinement scale $\Lambda_{B} \sim \mathrm{MeV}$. Then the condensate $\langle\bar{Q} Q\rangle$ induces the non-zero $\operatorname{VEV}\langle\chi\rangle \sim\langle\bar{Q} Q\rangle / M_{\chi}^{2}$, while for the majoron scale we have $f_{\beta} \sim \Lambda_{B}$. Let us also notice that due to the chiral anomaly of $U(1)_{B-L}$ current with respect to hidden gauge sector, the majoron $\beta$ will get a mass $\sim \Lambda_{B}$, becoming a sort of axion for the hidden sector related to its $\eta^{\prime}$ state. However, the mass of order $\mathrm{MeV}$ of $\beta$ cannot suppress $n \rightarrow$ $\tilde{n}+\beta$ transitions in nuclei.

\section{Discussion and outlook}

At this point, I am tempted to discuss a less orthodox idea, suggesting that the baryon number could be violated in the standard model itself, namely by the strong dynamics of the QCD sector. The conjecture is that the QCD itself could break baryon number by two units, by forming a six-quark condensate $\langle u d d u d d\rangle=\mathcal{B}=\Lambda_{B}^{9}$, along with the basic quark and gluon condensates, $\langle\bar{q} q\rangle$ and $\left\langle G^{2}\right\rangle$ or higher order operators $\langle\bar{q} G q\rangle,\langle\bar{q} q \bar{q} q\rangle$ which conserve baryon number. These sixquark condensates can be built upon different combinations of left and right $u, d$, and perhaps $s$ quarks, and they may have different convolutions of the Lorenz and color indices. Dynamically, they might be induced by the attractive forces between the electrically neutral three-quark trilinears $(u d d)_{8}$ in color-octet combinations, or as electrically neutral bound state of diquarks in color triplet combinations. ${ }^{9}$

\footnotetext{
9 With three quark flavors $u, d, s$, the condensate could appear in flavor singlet combination $\langle u d s u d s\rangle$ as a bound state of the three diquarks $u d$, $u s$, and $d s$. This would induce the Majorana mass term for the hyperon.
} 


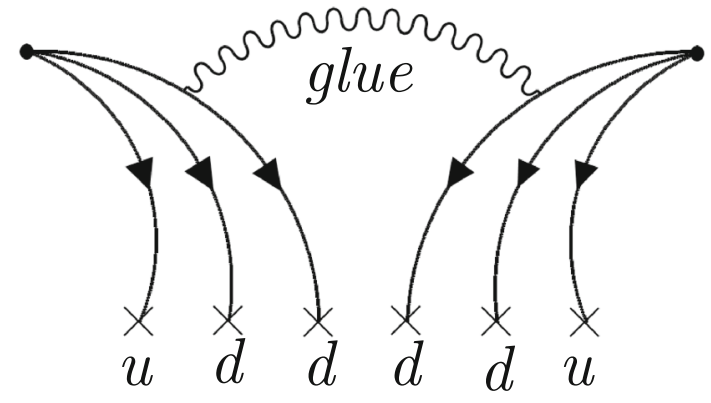

Fig. 4 Diagram generating the $n-\tilde{n}$ mixing via baryon-violating sixquark condensate $\langle u d d u d d\rangle$

A non-zero condensate $\langle u d d u d d\rangle$ would induce the neutron-antineutron mixing, as shown on Fig. 4. One can roughly estimate the mixing mass as $\epsilon_{n \tilde{n}} \sim \mathcal{B} /(1 \mathrm{GeV})^{8}$, simply taking scales of the neutron mass and residue and all relevant momenta order $1 \mathrm{GeV}$ and neglecting combinatorial numerical factors. Hence, for compatibility with the experimental limit, $\epsilon_{n \tilde{n}}<2.5 \times 10^{-24} \mathrm{eV}$, this condensate must be very fuzzy, with a mass parameter $\Lambda_{B}<1 \mathrm{MeV}$ or so. On the other hand, it is believed that any condensate in QCD, if it appears, must be characterized by the scale $\Lambda_{\mathrm{QCD}} \sim 100$ $\mathrm{MeV}$, as e.g. one has for the quark condensate $\langle\bar{q} q\rangle \sim \Lambda_{\mathrm{QCD}}^{3}$. Thus, we again encounter the problem of hierarchy, at least of 20 orders of magnitude, between the values $\Lambda_{B}^{9}$ and $\Lambda_{\mathrm{QCD}}^{9}$.

Formally, the theorem of Vafa and Witten [57] excludes the possibility of baryon number violating condensates in QCD. However, this theorem is based on assumptions which leave some loopholes. Namely, the proof of Ref. [57] is formally valid if all quarks are massive (in fact, one believes that all light quarks $u, d, s$ have masses of few $\mathrm{MeV}$ ), and, remarkably, if at the same time the vacuum angle $\Theta$ is exactly zero.

Therefore, one can envisage that in some imaginable world where the QCD vacuum angle is large, $\Theta \sim 1$, a baryonviolating condensate $\langle u d d u d d\rangle$ could exist, with $\mathcal{B} \sim \Lambda_{\mathrm{QCD}}^{9}$. In the absence of the axion mode which would relax $\Theta$ to zero, it could be formed as a dynamical reaction of the system tending to decrease the vacuum energy $\sim \cos ^{2} \Theta \Lambda_{\mathrm{QCD}}^{4}$ associated to non-zero $\Theta$. Thus, one can envisage that it value depends on the vacuum angle, $\mathcal{B}_{\Theta}=F(\Theta) \Lambda_{\mathrm{OCD}}^{9}$. According to the Vafa-Witten theorem, $\mathcal{B}$ should vanish in the limit $\Theta \rightarrow 0$, i.e. $F(0)=0$, while for $\Theta \sim 1$ one could have $\mathcal{B} \sim \Lambda_{\mathrm{QCD}}^{9}$. In addition, $\mathcal{B}_{\Theta}$ should be a periodic function of the vacuum angle, and it is natural to assume that it does not depend on the sign of $\Theta, F(\Theta)=F(-\Theta)$. These features are adequately described by a prototype function $F(\Theta)=C \sin ^{2} \Theta$, with $C$ being a constant $O(1)$.

In the real world, the vacuum angle might be non-zero: we have only an upper limit from the experimental searches of the electric dipole moment of the neutron, $\Theta<10^{-10}$ or so. Then the above estimation implies that the condensate is suppressed by a factor $\Theta^{2}<10^{-20}$, so that $\mathcal{B}=\Lambda_{B}^{9}=$ $C \Theta^{2} \Lambda_{\mathrm{QCD}}^{9}<C \times(1 \mathrm{MeV})^{9}$.

The baryo-majoron $\beta$ should emerge, as a composite Goldstone mode of this condensate, $\langle u d d u d d\rangle=$ $\mathcal{B} \exp \left(i \beta / f_{\beta}\right)$, with $f_{\beta} \sim \Lambda_{B}$, exactly like the pions emerge as the Goldstone modes of the quark condensate breaking the chiral $S U(2)_{L} \times S U(2)_{R}$ symmetry, $\langle\bar{q} q\rangle=$ $\Sigma \exp \left(i \tau_{a} \pi_{a} / f_{\pi}\right)$, with $\Sigma \sim \Lambda_{\mathrm{QCD}}^{3}$ and $f_{\pi} \sim \Lambda_{\mathrm{QCD}}$. The majoron coupling constant between $n$ and $\tilde{n}$ states is related to $\epsilon_{n \tilde{n}}$ via a Goldberger-Treimann-like relation, $g_{n}=\epsilon_{n \tilde{n}} / f_{\beta}$. Therefore, for $f_{\beta}<1 \mathrm{MeV}$, say with $\Lambda_{B} \simeq 200 \mathrm{keV}$, the nuclear stability limits concerning both the values of the mixing mass $\epsilon_{n \tilde{n}}$ and the Yukawa coupling $g_{n}$ can be respected.

An interesting feature of the dynamical baryon violation by the QCD can be that the order parameter $\Lambda_{B}$ could be different in vacuum and in dense nuclear matter, i.e. in nuclei or in the interiors of neutron stars. In particular, in dense nuclear matter spontaneous baryon violation could occur even if it does not take place in vacuum. Or right the opposite, dense nuclear matter could suppress the baryon-violating condensates. In this case, the search of neutron-antineutron oscillation with free neutrons and nuclear decay due to the neutronantineutron transition becomes a separate issue. Namely, it might be possible that the baryon-violating condensates evaporate at nuclear densities and do not lead to nuclear instabilities, while for free neutrons propagating in the vacuum they might be operational.

Acknowledgements The idea of this work emerged as a result of numerous discussions with Yuri Kamyshkov. I thank Yuri for motivating me to write it down and for a help in preparation of the manuscript. I would like to thank Gia Dvali, Oleg Kancheli, Arkady Vainshtein, and Andrea Addazi for many valuable discussions. The work was supported in part by the MIUR triennal grant for the Research Projects of National Interest PRIN 2012CPPYP7 "Astroparticle Physics".

Open Access This article is distributed under the terms of the Creative Commons Attribution 4.0 International License (http://creativecomm ons.org/licenses/by/4.0/), which permits unrestricted use, distribution, and reproduction in any medium, provided you give appropriate credit to the original author(s) and the source, provide a link to the Creative Commons license, and indicate if changes were made. Funded by SCOAP ${ }^{3}$.

\section{References}

1. E. Majorana, Il Nuovo Cimento 14, 171 (1937)

2. V.A. Kuzmin, Pisma. Zh. Eksp. Teor. Fiz. 12, 335 (1970)

3. R.N. Mohapatra, R.E. Marshak, Phys. Rev. Lett. 44, 1316 (1980) [Erratum-ibid. 44, 1643 (1980)]

4. K.S. Babu, R.N. Mohapatra, Phys. Lett. B 518, 269 (2001)

5. Z. Berezhiani, L. Bento, Phys. Rev. Lett. 96, 081801 (2006)

6. K.S. Babu et al., Phys. Rev. D 87, 115019 (2013)

7. K.A. Olive et al. [Particle Data Group Collaboration], Chin. Phys. C 38, 090001 (2014)

8. A.D. Sakharov, Pisma Zh. Eksp. Teor. Fiz. 5, 32 (1967) [JETP Lett. 5, 24 (1967)] 
9. A.D. Sakharov, Sov. Phys. Usp. 34, 392 (1991) [Usp. Fiz. Nauk 161, 61 (1991)]

10. V.A. Kuzmin, V.A. Rubakov, M.E. Shaposhnikov, Phys. Lett. B 155, 36 (1985)

11. Z. Berezhiani, A. Vainshtein, arXiv:1506.05096 [hep-ph]

12. S. Weinberg, Phys. Rev. Lett. 43, 1566 (1979)

13. S. Rao, R. Shrock, Phys. Lett. B 116, 238 (1982)

14. M. Baldo-Ceolin et al., Z. Phys. C 63, 409 (1994)

15. K.G. Chetyrkin, M.V. Kazarnovsky, V.A. Kuzmin, M.E. Shaposhnikov, Phys. Lett. B 99, 358 (1981)

16. J. Chung et al., Phys. Rev. D 66, 032004 (2002)

17. K. Abe et al. [Super-Kamiokande Collaboration], Phys. Rev. D 91, 072006 (2015)

18. D.G. Phillips II et al., Phys. Rept. 612, 1 (2016)

19. Y. Chikashige, R.N. Mohapatra, R.D. Peccei, Phys. Lett. B 98, 265 (1981)

20. R. Barbieri, R.N. Mohapatra, Z. Phys. C 11, 175 (1981)

21. G. Dvali, arXiv:hep-th/0507215

22. A. Addazi, Z. Berezhiani, Y. Kamyshkov, arXiv:1607.00348 [hep$\mathrm{ph}]$

23. M. Fukugita, T. Yanagida, Phys. Lett. B 174, 45 (1986)

24. G. Aad et al. [ATLAS Collaboration], Phys. Lett. B 754, 302 (2016)

25. Z.G. Berezhiani, Phys. Lett. B 129, 99 (1983)

26. Z.G. Berezhiani, Phys. Lett. B 150, 177 (1985)

27. Z. Berezhiani, A. Rossi, Nucl. Phys. Proc. Suppl. 101, 410 (2001)

28. A. Anselm, Z. Berezhiani, Nucl. Phys. B 484, 97 (1997)

29. Z. Berezhiani, Int. J. Mod. Phys. A 19, 3775 (2004)

30. Z. Berezhiani, Through the looking-glass: Alice's adventures in mirror world, eds. by M. Shifman et al. From Fields to Strings, vol. 3 (World Scientific, Singapore, 2005), pp. 2147-2195. arXiv:hep-ph/0508233

31. Z. Berezhiani, Eur. Phys. J. ST 163, 271 (2008)

32. G. Ban et al., Phys. Rev. Lett. 99, 161603 (2007)
33. A. Serebrov et al., Phys. Lett. B 663, 181 (2008)

34. I. Altarev et al., Phys. Rev. D 80, 032003 (2009)

35. K. Bodek et al., Nucl. Instrum. Meth. A 611, 141 (2009)

36. A. Serebrov et al., Nucl. Instrum. Meth. A 611, 137 (2009)

37. Z. Berezhiani, L. Bento, Phys. Lett. B 635, 253 (2006)

38. R.N. Mohapatra, S. Nasri, S. Nussinov, Phys. Lett. B 627, 124 (2005)

39. Y.N. Pokotilovski, Phys. Lett. B 639, 214 (2006)

40. Z. Berezhiani, Eur. Phys. J. C 64, 421 (2009)

41. Z. Berezhiani, A. Gazizov, Eur. Phys. J. C 72, 2111 (2012)

42. Z. Berezhiani, F. Nesti, Eur. Phys. J. C 72, 1974 (2012)

43. Z. Berezhiani, arXiv:1602.08599 [astro-ph.CO]

44. Z. Berezhiani, M.I. Vysotsky, Phys. Lett. B 199, 281 (1987)

45. Z. Berezhiani, A.Y. Smirnov, Phys. Lett. B 220, 279 (1989)

46. H.M. Georgi, S.L. Glashow, S. Nussinov, Nucl. Phys. B 193, 297 (1981)

47. M. Doi, T. Kotani, E. Takasugi, Phys. Rev. D 37, 2575 (1988)

48. Z. Berezhiani, A.Y. Smirnov, J.W.F. Valle, Phys. Lett. B 291, 99 (1992)

49. A. Gando et al. [KamLAND-Zen Collaboration], Phys. Rev. C 86, 021601 (2012)

50. K. Choi, A. Santamaria, Phys. Rev. D 42, 293 (1990)

51. Z. Berezhiani, G. Fiorentini, M. Moretti, A. Rossi, Z. Phys. C 54, 581 (1992)

52. Z. Berezhiani, M. Moretti, A. Rossi, Z. Phys. C 58, 423 (1993)

53. Z. Berezhiani, A. Rossi, Phys. Lett. B 336, 439 (1994)

54. L. Bento, Z. Berezhiani, Phys. Rev. D 64, 115015 (2001)

55. L. Bento, Z. Berezhiani, Phys. Rev. D 62, 055003 (2000)

56. G.G. Raffelt, Stars as Laboratories for Fundamental Physics (Chikago University, Press, 1996)

57. C. Vafa, E. Witten, Nucl. Phys. B 234, 173 (1984) 